\title{
Effect of Graphene for Ablation Study of Advanced Composite Materials for Aerospace Applications
}

\author{
SADIA Sagar lqbal ${ }^{1, a,{ }^{*}}$, ANEELA Sabir ${ }^{1, b}$, ATIF Islam ${ }^{1, c}$, \\ SYED Zain-ul-Abdeen Bukhari ${ }^{2, d}$, MUHAMMAD Yasir ${ }^{3, e}$, \\ ARSHAD Bashir ${ }^{4, f}$, ALI Bahadar ${ }^{5,9}$ \\ ${ }^{1}$ Department of Polymer Engineering \& Technology, CEET, University of the Punjab, Pakistan \\ ${ }^{2}$ Quality Enhancement Cell, University of the Punjab, Pakistan \\ ${ }^{3}$ Department of Materials Sciences and Engineering, \\ Institute of Space Technology, Islamabad-44000, Pakistan \\ ${ }^{4}$ Institute of Industrial Control System, P.O. Box 1398, Rawalpindi, Pakistan \\ ${ }^{5}$ Department of Chemical and Materials Engineering, \\ King Abdulaziz University, Rabigh, Saudi Arabia

\begin{abstract}
asadia.pet.ceet@pu.edu.pk, baneela.pet.ceet@pu.edu.pk, 'dratifislam@gmail.com, dzainisyed8382@gmail.com, êmuhammadyasir85@gmail.com, fhmarshid@yahoo.com, gengrbahadur@gmail.com
\end{abstract}

Keywords: Graphene, Styrene butadiene rubber, Ablation testing, Thermal conductivity, Aerospace

\begin{abstract}
Graphene was incorporated into elastomeric Matrices using dispersion kneader and two roller mixing mill to fabricate ablative nanocomposites used in hyper-thermal environment encountered by space vehicle or rocket motor. The addition of graphene in the host matrix has remarkably reduced the back face temperature elevation during the ablation testing of the ablatives. The linear and mass ablation resistances have been diminished while insulation indexes of the nanocomposites have been increased the graphene incorporation into the elastomeric matrix. Thermal stability and heat absorbance capability of the polymer nanocomposites were progressed with increasing the filler to matrix ratio. Thermal conductivity of the ablatives have been conducted according to the ASTM E1225-99 and D5470-03, respectively to execute the effect of graphene concentration on the thermal transport characteristics of the tested specimens. Tensile strength of the nanocomposite specimen was augmented with increasing graphene to polymer ratio. Scanning electron microscopy was used to scrutinize the evenly dispersed graphene in the polymer matrix, polymer pyrolysis, and voids formation in the ablated nanocomposites.
\end{abstract}

\section{Introduction}

Polymer nanocomposite is a combination of a polymer matrix and nano-reinforcement. Thermal, mechanical and electromagnetic properties of PNC are quite different compared to the pristine polymer matrix[1]. Nano-incorporations like nanofillers, nanotube, nanofibers and nanorods interact with the molecular chains of the polymer chemically or physically at the nanoscale which remarkably influences the physical and chemical properties of the host matrix. Styrene butadiene rubber (SBR) is a copolymer of styrene and butadiene. Styrene butadiene rubber (SBR) is produced using emulsion or solution polymerization. SBR composites are used in tire treads, conveyor belt covers, gaskets, mats, shoe soles, etc due to its excellent impact strength, good resilience, tensile strength, abrasion resistance, metallic adhesion, tear resistance, acid resistance, water resistance and maintains flexibility at lower temperatures. SBR is a thermoplastic rubber, crosslinked with the addition of a crosslinker i.e. sulphur, peroxides or radiation crosslinking, etc. A crosslinked rubber has better thermal stability, mechanical strength, aging resistance, abrasion resistance, weather resistance and tear strength compared to the pristine rubber. Different fillers (nanoclays, nanocarbon, nanosilica, carbon nanotubes, graphene, silicon carbide, alumina, etc) have been used 
to tailor the thermal, mechanical, adhesion, abrasion, etc properties of the SBR [2]. SBR is widely used in tire industry. During the vehicle operation, heat produced due to the friction of tire surface with road that may degrade the mechanical strength of the rubber. So, in order to sustain the mechanical strength of tire thermal endurance of the rubber should be compatible. Graphene are believed to be remarkable filler for polymer matrixes due to its promise-able characteristics [3]. Graphene is one of the rapidly emerging filler used in polymeric systems to fabricate polymer nanocomposites that are utilized in aerodynamic, sports, membranes, electrical, electronic, etc., industries due to light weight, high strength, and better thermal stability compared to the pristine polymer matrices, counterpart. The most important feature is thermal properties i.e. thermal conductivity/diffusivity, thermal impedance, thermos-gravimetric stability, glass/crystallization/melting temperatures and their enthalpies have great importance for polymer nanocomposites because they define their suitable area of application. The novelty of this research paper is effect of various concentration of graphene on the ablation, thermal degradation, heat absorbing capability, thermal conductivity and mechanical characteristics of the SBR nanocomposites.

\section{Experimental}

Materials. Graphene (fabricated through CVD method with Fe catalyst, Purity $>95 \%$, diameter 20$30 \mathrm{~nm}$, and length 50-70 $\mathrm{m}$, average aspect ratio 2400:1) were supplied by Nanoport Co. Ltd, China and they were used as received. Emulsified Styrene butadiene rubber (INDOPOL 1502) was purchased from Evergreen Global Pte. Singapore. Nanocarbon, Sulphur, Zinc oxide, wax and stearic acid were purchased from BDH. Vulcanizing accelerators MBTS and HBS were purchased from Dalian Richon Chemical Co. Ltd, China. Aromatic oil was purchased from International petrochemicals (Pvt) Ltd, Pakistan.

Formulation and Fabrication of Graphene/SBR Nanocomposites. Graphene along with the nanocarbon (reinforcing filler), cross linker (Sulphur), accelerators (MBTS \& HBS), activators (zinc oxide, stearic acid), and plasticizers (aromatic oil, wax) were incorporated in the SBR matrix according to the following formulation presented in Figure 1. Graphene and nanocarbon were dispersed in the polymer matrix using dispersion kneader at $110^{\circ} \mathrm{C}$ for 30 minutes and then other processing aids were incorporated in the filled rubber sheet on two roller mixing mill at $60^{\circ} \mathrm{C}$ and 50rpm roller's speed for 20 minutes. Two types of ablative composite specimens according to ASTM E285-08 were fabricated on the hot isostatic press at $140^{\circ} \mathrm{C}$, and $1500 \mathrm{psi}$ for 50 minutes, that were $100 \times 100 \times 10 \mathrm{~mm}^{3}$ (P ablator) and $100 \times 100 \times 10 \mathrm{~mm}^{3}$ with $10 \mathrm{~mm}$ diameter cavity in the center of the specimen ( $\mathrm{R}$ ablator). $\mathrm{P}$ ablators were used to investigate the linear ablation resistance and backface temperature evolution during the ablation testing in head on impingement (HOI) mode of oxy-acetylene $(\mathrm{O}-\mathrm{A})$ torch flame. Radial/mass ablation rates and \% char yield were measured for $\mathrm{R}$ ablated specimens i.e. ablated in parallel flow (PF) of $\mathrm{O}-\mathrm{A}$ flame trough the $10 \mathrm{~mm}$ cavity. HOI is meant to simulate the direct impingement of shock waves or flame fronts on the aerodynamic/included surfaces in reentry or propulsion modules. While PF configuration simulates the shear effects generated on the bounding walls of flow such as encountered at the aft-end and nozzle throat of propulsion modules. Similar process was adopted to fabricate the Composite specimens with $6 \times 6 \times 0.12$ inch $^{3}$ dimensions and tensile testing samples according to ASTM D412-98A and for each turn six samples were collected. Five diverse concentrations of Graphene, i.e., $0,0.1,0.3,0.5$, and $1 \mathrm{wt} \%$ were reinforced in the rubber matrix, separately to fabricate polymer nanocomposite specimens that are nominated as S10, S11, S12, S13 and S14, respectively.

Characterization of Graphene/SBR Nanocomposites. Ablation testing of $\mathrm{P} \& \mathrm{R}$ ablators was carried out using ASTM 285-08 in HOI and PF modes of O-A flame. Experimental setup for the anti-ablation study of ablative nanocomposites is demonstrated in Figure 1. O-A torch was used as a high temperature source (Flame temperature $\sim 3000^{\circ} \mathrm{C}$ with heat flux of $8 \times 10^{6} \mathrm{~W} / \mathrm{m}^{2}$ measured with pyrometer IRAH35 U, Japan) i.e. exposed on the central front face of the P ablators and the flame let to pass through the $10 \mathrm{~mm}$ cavity in case of $\mathrm{R}$ ablators. The flow rate of both Oxygen and acetylene gases was maintained at $0.35 \mathrm{~m}^{3} / \mathrm{h}$ and their pressures were $50 \mathrm{psi}$ and $23 \mathrm{psi}$, respectively 
during the ablation testing. The torch was kept at $10 \mathrm{~mm}$ far from the $\mathrm{P}$ ablator surface. Three thermocouples ( $\mathrm{K}$ type) having temperature sensing range -200 to $1350^{\circ} \mathrm{C}$, were used at the backface central region of the $\mathrm{P}$ ablator. These thermocouples linked with the data logger TECPEL 319 , also connected to the laptop with RS-232 data cable. Time-temperature online contours for a specific duration were established on the laptop screen during the O-A torch exposure on the $\mathrm{P}$ ablator's front face. All equipment used for the current investigations are calibrated prior to their utilization according to ASTM standards. Figure 1 simulates the ultrahigh temperature oxyacetylene flame plumes in full bloom in head on impingement and shear modes during the ablation testing of the ablative composite.

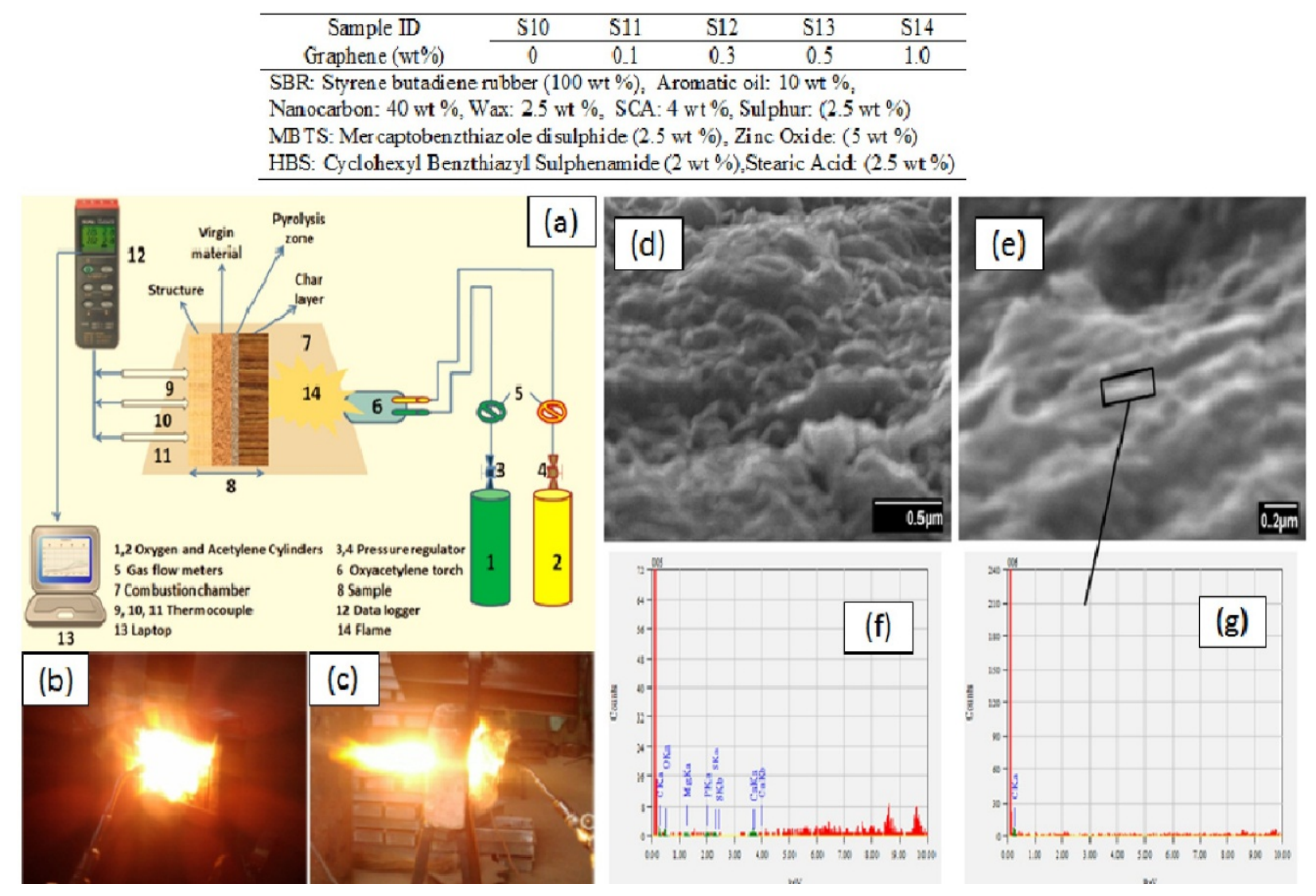

Fig. 1. Basic formulation with different wt. \% loadings of Graphene, schematic illustration of the experimental setup for ablation testing (a), impingement (b) shear (d) modes photographs during the ablation testing, and SEM images of graphene distribution in the SBR matrix $(\mathrm{d}, \mathrm{e})$ and the compositional analysis of S5 nanocomposite (f) and a graphene ( $\mathrm{g}$ ) embedded in the composite of the ablative composite

Linear/radial/mass ablation rates and \% char yield for P \& $\mathrm{R}$ ablators were measured according to the following formulae [4]. Scanning electron microscopy (SEM, JSM 6940A, Jeol, Japan) along with the energy dispersive spectroscopy was used to observe the dispersion of MWNTs in the SBR matrix, surface analysis of the post thermal conductivity tested specimens, compositional analysis of the nanotube and nanocomposite. Perkin Elmer Diamond $100 \mathrm{TG} / \mathrm{DTA}$, Japan was used to analyze the thermal degradation and endothermic/exothermic response of the polymer nanocomposites within the temperature span $25-1000^{\circ} \mathrm{C}$ at a heat integrate viz. $10^{\circ} \mathrm{C} / \mathrm{min}$ in ambient atmosphere at my present institute. Thermal conductivity $\left(\lambda_{N}\right)$ of the nanocomposite specimens were carried out using ASTM E1225-99. The tested specimen has 1 inch $^{2}$ area and $3 \mathrm{~mm}$ thickness. Copper was used as a relativistic material for the measurement of $\lambda_{N}$. Tensile testing of the nanocomposite specimens was carried out on universal tensile testing machine (AG-20KNXD Plus, Shimadzu) according to ASTM standard D412-98A to evaluate ultimate tensile strength, elongation at break, elastic modulus and plasticity of the polymer composite specimens. Shore A hardness of the rubber composites was examined on Torsee, Tokyo testing machine to simulate the effect of the variant reinforcements and their loading concentrations on the fabricated elastomeric specimen hardness. 


\section{Results and Discussion}

Dispersion of Graphene in Styrene Butadiene Rubber. SEM coupled with EDS was performed to investigate the morphology/compositional analysis of graphene reinforced SBR nanocomposites. Figure $1(\mathrm{~d}, \mathrm{e}, \mathrm{f}, \mathrm{g})$ elucidates the even dispersion of graphene in the host rubber matrix along with the compositional analysis of the graphene. The homogenous dispersion of graphene in the polymer matrix is due to the proper diffusion of graphene into the rubber matrix in the heating environment of internal dispersion kneader. The longitudinal, transverse and horizontal flow of material during its passage through the twin-roll nip of two-roller mixing mill in the post mixing stage also imparts triaxial flow to disperse graphene efficiently in the SBR matrix.

Ablation Testing. The backface temperature profiles of the composite specimens were recorded during the ablation testing to investigate the capability of the polymer nanocomposites to withstand hyperthermal environment. The effect of graphene to reduce the backface temperature, peak backface temperature elevation, and backface temperature evolution rate of the rubber nanocomposites, is elaborated in Figure $2 \mathrm{a}$ and $2 \mathrm{~b}$. The $1 \mathrm{wt} \%$ addition of graphene in the SBR matrix has efficiently enhanced the efficiency of the polymer nanocomposite to reduce the peak backface temperature elevation up to $31 \%$ and backface temperature evolution rate up to $38 \%$ due to the excellent thermal stability, heat absorbing capability, nanoscale interaction with the polymeric chains, and the even dispersion of graphene in the rubber matrix [5]. Insulation index $\left(\mathrm{I}_{\mathrm{T}}\right)$ of a material defines the capability of the specimen that how longer it can survive in the extreme heating environment. $\mathrm{I}_{\mathrm{T}}$ of the composite specimens were measured from the time-temperature contours of the polymer nanocomposite by using the formulae and the effect of graphene concentration on the $I_{T}$ of the rubber nanocomposites is displayed in Figure $2 c$. It is observed that $I_{T}$ reduces with increasing filler contents in the rubber matrix. The $1 \mathrm{wt} \%$ successful incorporation of the graphene into the host matrix has raised $\mathrm{I}_{\mathrm{T}}$ of the rubber nanocomposite up to 2225,4342 , and $7039 \mathrm{~s} / \mathrm{m}$ at 60,80 , and $100^{\circ} \mathrm{C}$ backface temperatures, respectively relative to the base composite formulation S10, counterpart. Linear/mass ablation rates and \% char yield of the post burnt ablated composite specimens are depicted in Figure 3a. It is observed that all three parameters are reduced simultaneously with increasing graphene concentration in the polymer matrix. The $1 \mathrm{wt} \%$ addition of graphene in the rubber matrix has remarkably augmented the linear ablation resistance up to $46.5 \%$; mass ablation resistance elevated up to $41.2 \%$; and effectively reduced the $\%$ char yield of the ablative nanocomposites up to $6.4 \%$ relative to the base composite formulation, S10. The minimum ablation rate is noticed for S14, i.e., $0.046 \mathrm{~mm} / \mathrm{s}$ due to the high thermal stability, efficient heat quenching capability, and even dispersion of the graphene in the SBR matrix along with the vaporization induced heat quenching by the aromatic liquid. Oxy-acetylene torch flame was passed through the $10 \mathrm{~mm}$ diameter hole of the ablative nanocomposites to scrutinize the effect of graphene loading concentration on the radial/mass ablation resistance and $\%$ char yield of composite specimens in the shear flow of the hot stream of gases. Figure $3 \mathrm{~b}$ elucidates that radial ablation rate is reduced from 0.25 to $0.15 \mathrm{~mm} / \mathrm{s}$; mass ablation rate is diminished from 0.19 to $0.07 \mathrm{~g} / \mathrm{s}$; and percent char yield declined from 10.93 to $4.42 \%$ with the $1 \mathrm{wt} \%$ incorporation of the graphene into the base composite formulation. The least radial ablation rate $(0.15 \mathrm{~mm} / \mathrm{s})$ is measured for S14 due to excellent thermal endurance and uniform dispersion of graphene in the host polymer matrix[6]. 


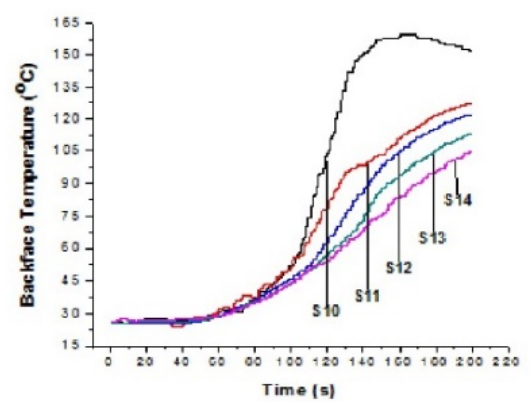

(a)

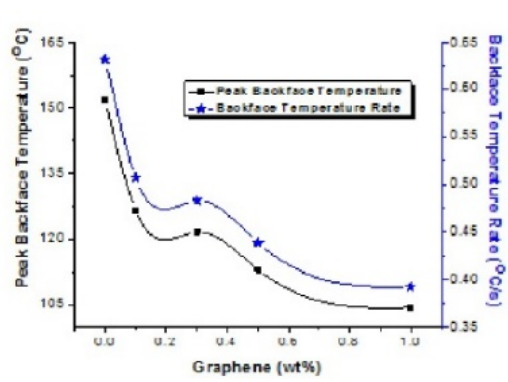

(b)

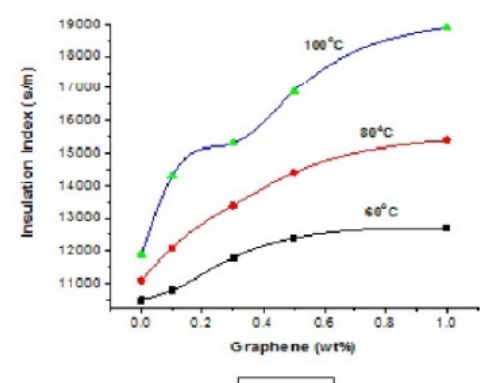

(c)

Fig. 2. Backface temperature profiles (a) peak backface temperature and backface temperature evolution rates (b), and Insulation indexes (c) recorded during the ablation testing on the oxy-acetylene torch of the fabricated ablative nanocomposites
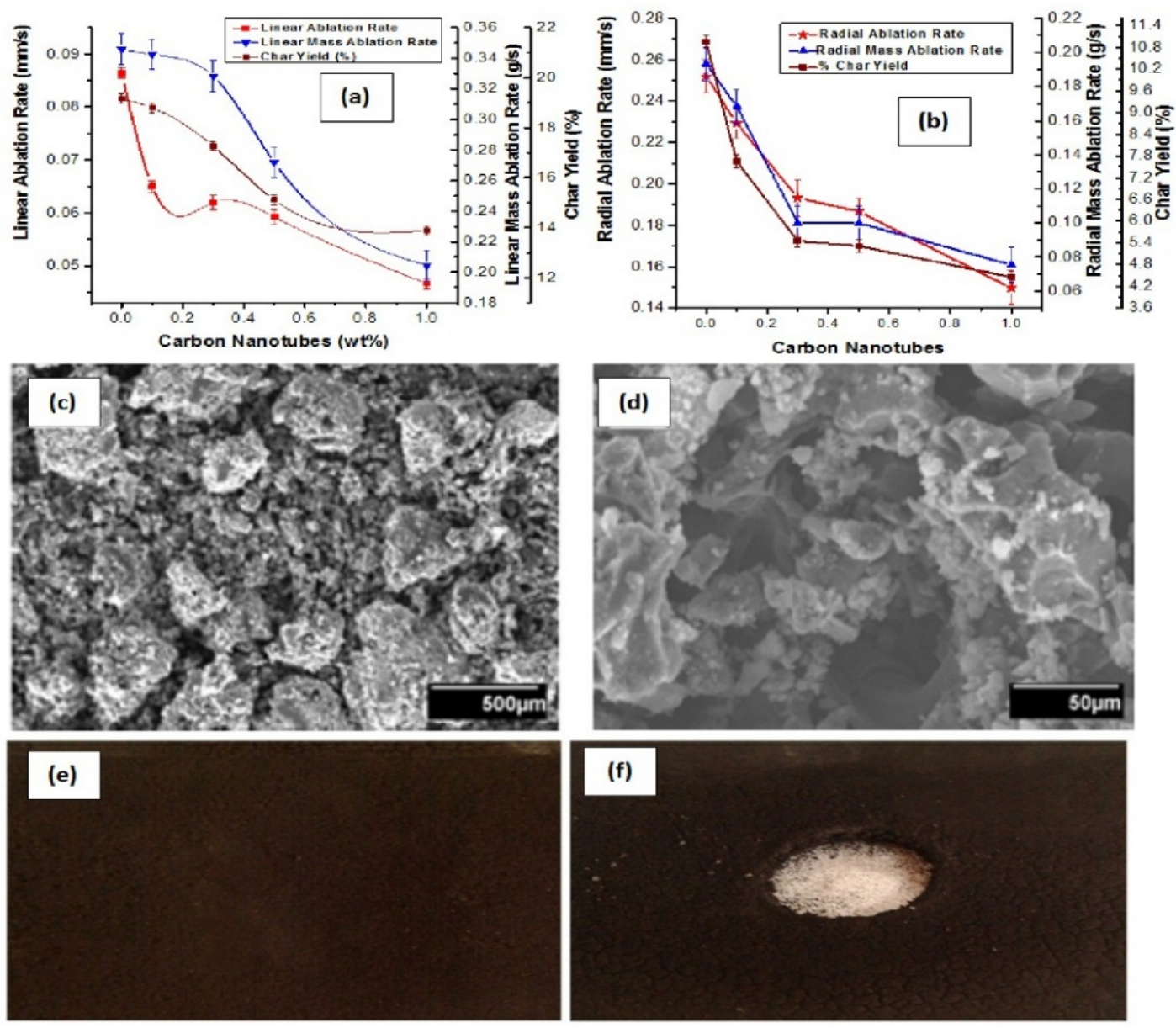

Fig. 3. Linear/Radial ablation-mass ablation rates and \% char yield (a, b), SEM of ablated char morphology (c, d), and photographs (e, f) of the post ablation tested composite specimens

The ablated sample's char morphology and its compositional analysis are displayed in Figure 3(c d). The porous structure was developed during the ablation test which enhanced the transpirational and evaporation processes that reduced the backface temperature elevation. Major elements found in the EDS analysis of the S14 ablative nanocomposite are C, S, Si, O and Zn. Figure $3(\mathrm{e}, \mathrm{f})$ simulates that the ablative nanocomposite is ablated in patches not continuously which is not favorable to reduced mechanical erosion resistance in hyperthermal environments[6]. These patches can easily be removed with high velocity hot gases flow during the ablation testing of the composite specimens and as a result the ablation rates also increases. The observed morphology explains why SBR nanocomposites have the least ablation resistance compared to silicon rubber, NBR, and EPDM nanocomposites. 
Thermal Properties. Thermo-gravimetric analysis of the rubber nanocomposites in the 25 to $800^{\circ} \mathrm{C}$ temperature is depicted in Figure 4a to ascertain the effect of the graphene loadings on the thermal stability of SBR matrix. Insignificant thermal decomposition is observed up to $460^{\circ} \mathrm{C}$ and the maximum thermal oxidation of the composite specimens is noticed in the subsequent $140^{\circ} \mathrm{C}$ temperature scan. Thermal stability of the polymer nanocomposites has been elevated with increasing graphene concentration in the rubber matrix as is clear from Figure 4a that illustrates the comparative study of weight loss at four diverse temperatures due to the efficient thermal endurance and uniform dispersion of the graphene in the rubber matrix. Moreover, durable filler-to-matrix interaction due to the presence of SCA in the SBR matrix plays an important role to diminish the thermal decomposition of the fabricated ablative composites[7].

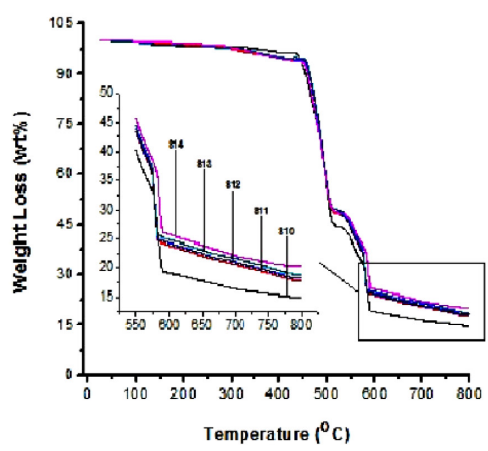

(a)

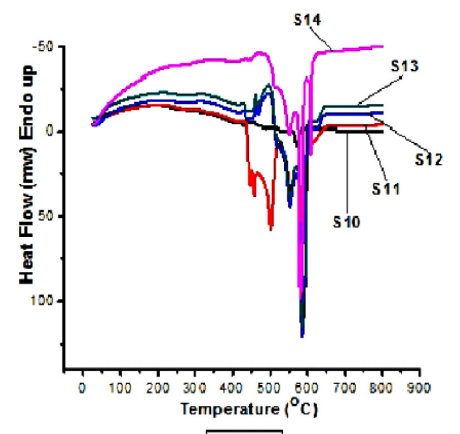

(b)

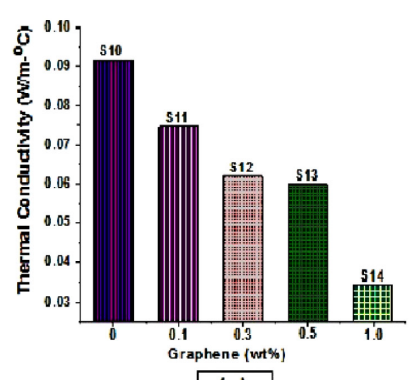

(c)

Fig. 4. Effect of graphene concentrations on the thermal degradation (a), heat flow response (b), and thermal conductivity (c) of polymer nanocomposites

Figure $4 \mathrm{~b}$ elucidates the heat flow response of the polymer nanocomposites and the maximum variation in heat flow is observed in the temperature range in which the maximum thermal degradation has been noticed in the thermos-gravimetric analysis. The composite materials tend towards the endothermic response with the progressive incorporation of graphene in the host matrix. The composite specimen S14 ablator has absorbed the maximum input heat compared to other ones due to the phonon capturing within the incorporated graphene, other factors remaining the same[8, 9]. Figure $4 \mathrm{c}$ illustrates the effect of graphene on the thermal conductivity of SBR rubber nanocomposites. It is observed that with increasing the graphene concentration in the polymer matrix, thermal resistivity of the nanocomposite specimens has outstandingly enhanced. The $1 \mathrm{wt} \%$ impregnation of graphene has improved the thermal resistivity of SBR nanocomposite up to $64 \%$ due to the heat dissipation along and in chiral direction of the incorporated graphene. The minimum thermal conductivity was observed for $\mathrm{S} 14$ i.e. $0.033 \mathrm{~W} / \mathrm{m}-{ }^{\circ} \mathrm{C}$. Thermal transport through the solids is mainly attributed due to the phonons transport through the body. During the heat transport through the graphene/SBR nanocomposites, phonons are entrapped within the nanotube and their network. Consequently, thermal conductivity of the nanocomposite is remarkably reduced with increasing graphene to matrix ratio[8, 10, 11].

Mechanical Testing. Universal tensile testing machine was used to execute the stress-strain behavior of the fabricated ablative nanocomposites. Figure 5a illustrates that with increasing filler concentration in the rubber matrix, stress the bearing capability of the nanocomposites is also progressed without suffering elongation at break. Ultimate tensile strength and elongation at break of the composite specimens were measured from the stress-strain contours and are depicted in Figure $5 \mathrm{~b}$ [12]. It is observed that ultimate tensile strength is raised up to $56 \%$ and elongation at break declined up to $13 \%$ with the $1 \mathrm{wt} \%$ incorporation of the graphene into base composite formulation showing excellent mechanical strength, strong filler-matrix bonding and uniform dispersion of the incorporated graphene into the rubber matrix. Figure 5c illustrates the effect of graphene concentration on the rubber hardness of the ablative nanocomposites. The graph simulates that the progressive graphene impregnation in the rubber matrix has augmented the rubber hardness of S14 up to 60 shore A relative to the base composite formulation (S10) i.e. 50 shore A, 
counterpart. This improvement in the rubber hardness of the polymer nanocomposites is due to ultrahigh elastic modulus, nanoscale interaction with the polymer chains, and the uniform dispersion of graphene in the rubber matrix $[9,13]$.
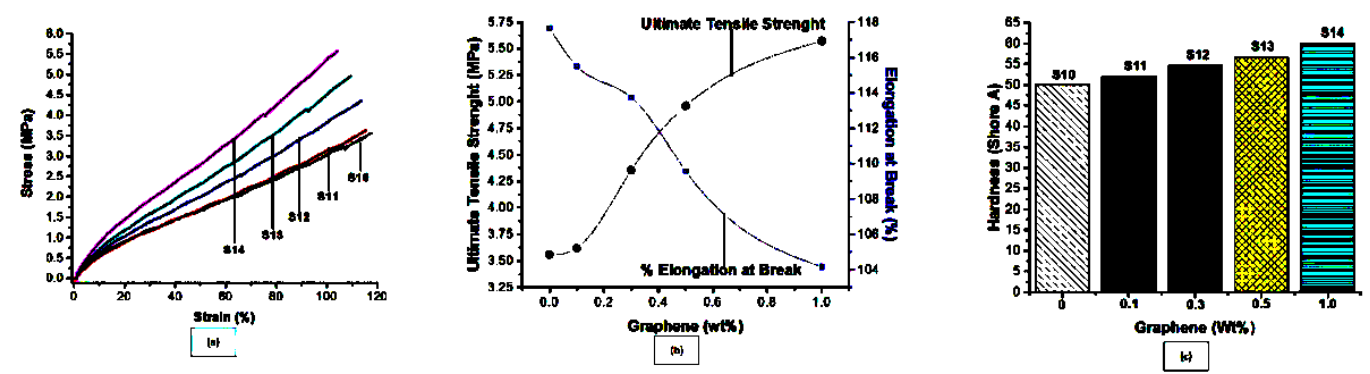

Fig. 5. Stress-strain contours (a), ultimate tensile strength and elongation at break behavior (b), and Shore A rubber hardness (c) of the ablative nanocomposites with diverse graphene impregnation in the SBR matrix

\section{Conclusion}

Graphene were uniformly dispersed in the SBR rubber matrix using dispersion kneader and two-roller mixing mill. The progressive incorporation of graphene into the polymer matrix has remarkably enhanced the anti-ablation performance of the nanocomposites. Linear/mass ablation resistance have been improved up to 46.5 and $41.2 \%$ while percent char yield is reduced up to $6.4 \%$; peak backface temperature during the ablation test has been diminished up to $38 \%$ while insulation index has been enhanced up to $58 \%$ at $100^{\circ} \mathrm{C}$ back face temperature with the $1 \mathrm{wt} \%$ addition of graphene in the host matrix. Thermal stability and heat quenching capability of the polymer nanocomposites have been elevated with increasing graphene loading concentration in the rubber matrix. Thermal conductivity of the nanocomposites was reduced with increasing the graphene to matrix ratio due to the phonons entrapping and heat dissipation within the uniformly dispersed graphene network in the elastomeric matrix. Ultimate tensile strength and Shore A rubber hardness of the rubber composites have been efficiently improved while elongation at break is reduced with increasing filler to matrix ratio. The SBR composite shows spelling following exposure to ultrahigh temperatures and shear flow in contrast to silicone, NBR, and EPDM nanocomposites.

\section{Acknowledgement}

The authors would like to greatly acknowledge Research Grant Fiscal Year (2017-2018), University of the Punjab, Lahore, Pakistan for providing sufficient funds to execute this research, School of Chemical and Materials Engineering (SCME), National University of Sciences and Technology (NUST), and Longman Mills, Lahore to aid us with the best of their elastomeric composites fabrication and testing expertise and facilities.

\section{References}

[1] M. Moniruzzaman, K.I. Winey, Polymer nanocomposites containing carbon nanotubes, Macromolecu., 39 (2006) 5194-5205.

[2] H. Ma, L. Tong, Z. Xu, Z. Fang, Synergistic effect of carbon nanotube and clay for improving the flame retardancy of ABS resin, Nanotechnol., 18 (2007) 375602.

[3] M.A. Atieh, Effect of Functionalized Carbon Nanotubes with Carboxylic Functional Group on the Mechanical and Thermal Properties of Styrene Butadiene Rubber, Fuller. Nanotub. Carbon Nanostruct., 19 (2011) 617-627.

[4] N. Iqbal, S. Sagar, M.B. Khan, H.M. Rafique, Ablation, thermal stability/transport and mechanical investigations of modified nanokaolinite impregnated acrylonitrile butadiene rubber composites, J. Comp. Mater., 48 (2013) 1221-1231. 
[5] J.K. Park, T.J. Kang, Thermal and ablative properties of low temperature carbon fiber-phenol formaldehyde resin composites, Carbon. 40 (2002) 2125-2134.

[6] N. Iqbal, S. Sagar, M.B. Khan, M.I. Bassyouni, Z.M. Khan, Aluminum silicate fibers impregnated acrylonitrile butadiene rubber composites: Ablation, thermal transport/stability, and mechanical inspection, J. Appl. Poly. Sci., 130 (2013) 4392-4400.

[7] S.S. Iqbal, F. Inam, A. Bahadar, M.A. Bashir, F. Hassan, M.B. Khan, Z.M. Khan, T. Jamil, Ablation, thermal stability/transport/phase transition study of carbon nanofiber-reinforced elastomeric nanocomposites, J. Therm. Analy. Calorim., (2017) 1-10.

[8] S. Sagar, N. Iqbal, A. Maqsood, U. Javaid, Thermogravimetric, differential scanning calorimetric and experimental thermal transport study of MWCNT/NBR nanocomposites, J. Therm. Analy. Calorim. 114 (2013) 161-167.

[9] N. Iqbal, S. Sagar, M.B. Khan, Comprehensive ablation characteristics of ceramic fibers impregnated rubber composites, Intern. J. Eng. Technol., 6 (2014) 162-167.

[10] S.S. Iqbal, N. Iqbal, T. Jamil, A. Bashir, Z.M. Khan, Tailoring in thermomechanical properties of ethylene propylene diene monomer elastomer with silane functionalized multiwalled carbon nanotubes, J. Appl. Poly. Sci., 26 (2015) 232-239.

[11] S. Sagar, N. Iqbal, A. Maqsood, Multiwalled carbon nanotubes impregnated rubber nanocomposites: thermal transport/decomposition and differential scanning calorimetric study, J. Reinfor. Plast. Compo., 32 (2013) 1052-1061.

[12] N. Iqbal, S. Sagar, M.B. Khan, H.M. Rafique, Elastomeric ablative nanocomposites used in hyperthermal environments, Poly. Eng. Sci., 54 (2013) 255-263.

[13] F. Jamshaid, A. Ahmad, M. Adrees, S.S. Iqbal, H. Zaheer, T. Jamil, J. Ahmad, T. Hussain, Tuning the interlaminar shear strength and thermo-mechanical properties of glass fiber composites by incorporation of (3-mercaptopropyl) trimethoxysilane-functionalized carbon black, Iran. Poly. J., 11 (2017) 1-15. 